\title{
Long-term Outcome and Efficacy of Cyclophosphamide Therapy in Japanese Patients with ANCA-associated Microscopic Polyangiitis: A Retrospective Study
}

\author{
Izaya Nakaya ${ }^{1}$, Mayumi Yahata ${ }^{1}$, Satoko Takahashi ${ }^{1}$, Tomomi Sasajima ${ }^{2}$, \\ Tsutomu Sakuma ${ }^{3}$, Yugo Shibagaki ${ }^{4}$ and Jun Soma ${ }^{1}$
}

\begin{abstract}
Objective The aim of this study was to elucidate the efficacy of cyclophosphamide (CY) in Japanese patients with antineutrophil cystoplasmic antibody (ANCA)-associated microscopic polyangiitis (MPA).

Methods Sixty-four patients, newly diagnosed with ANCA-associated MPA were included in this retrospective study. The patients were divided into two groups based on whether they received combination therapy of CY and corticosteroid (CS) (CY group, n=29) or CS alone (CS group, $n=35$ ) for remission induction. The primary outcome was all-cause mortality.

Results Most patients in the CY group were treated with oral CY. Between the two groups, there were no differences in the baseline characteristics except for a higher proportion of male patients in the CY group. The remission rate was not substantially different between the two groups $(86.2 \%$ in the CY group vs. $91.4 \%$ in the CS group). The survival rate was slightly higher in the CY group than in the CS group (not statistically significant; 0.86 vs. 0.77 at 1 year and 0.73 vs. 0.64 at 5 years, $p=0.648$ ). In the $C Y$ group, the hazard ratio after adjusting for age, sex, Birmingham vasculitis activity score values, serum albumin levels and Creactive protein (CRP) levels was 0.657 (95\% CI, 0.254-1.699; $\mathrm{p}=0.386$ ).

Conclusion We observed no increased efficacy of CY in ANCA-positive MPA in the Japanese patients, and hence, its efficacy may be limited in these patients.
\end{abstract}

Key words: anti-neutrophil cytoplasmic antibody, Birmingham vasculitis activity score, cyclophosphamide, microscopic polyangiitis, remission induction

(Intern Med 52: 2503-2509, 2013)

(DOI: 10.2169/internalmedicine.52.0199)

\section{Introduction}

Antineutrophil cytoplasmic antibody (ANCA)-associated vasculitis (AAV) is classified into four types: granulomatosis with polyangiitis (GPA), eosinophilic granulomatosis with polyangiitis, microscopic polyangiitis (MPA) and the renal limited variant of MPA (RLV) (1).

The clinical background of AAV in Japan has recently been reported to be different from that in Europe. First, proteinase-3 (PR3)-ANCA-associated GPA is uncommon in
Japan, whereas myeloperoxidase (MPO)-ANCA-associated MPA constitutes a large proportion of Japanese AAV cases (2-4). Second, in Europe, the use of a combination of corticosteroid (CS) and cyclophosphamide (CY) for remission induction in AAV has markedly improved patient survival, and it remains the mainstay of AAV treatment to date (5-7). However, the efficacy of CY has not yet been studied in Japanese patients with AAV. In a Japanese nationwide survey of rapidly progressive glomerulonephritis (RPGN) cases, approximately half of which were AAV cases, the number of deaths due to infections exceeded that

\footnotetext{
${ }^{1}$ Department of Nephrology, Iwate Prefectural Central Hospital, Japan, ${ }^{2}$ Department of Respiratology, Iwate Prefectural Central Hospital, Japan, ${ }^{3}$ Department of Pathology, Iwate Prefectural Central Hospital, Japan and ${ }^{4}$ Division of Nephrology and Hypertension, St. Marianna University School of Medical Hospital, Japan
}

Received for publication January 21, 2013; Accepted for publication June 30, 2013

Correspondence to Dr. Izaya Nakaya, inakaya@chuo-hp.jp 
caused by the primary disease $(8,9)$. Therefore, the combination treatment with $\mathrm{CY}$ and CS is uncommon in Japan because of the high risk of life-threatening infections (8). Moreover, few studies have investigated the long-term prognosis and actual causes of death in Japanese patients with AAV $(8,9)$. The aim of this study was to clarify the longterm outcome and efficacy of $\mathrm{CY}$ for remission induction in Japanese patients with ANCA-positive MPA.

\section{Materials and Methods}

\section{Ethics}

This study was approved by the ethics committee of Iwate Prefectural Central Hospital and was conducted in accordance with the ethical principles of the Declaration of Helsinki. We did not obtain written informed consent from each participant because the ethical guidelines for epidemiological research in Japan did not require consent for a retrospective study in which the researchers reviewed only the medical records (10).

\section{Participants}

We screened 81 consecutive Japanese patients who were newly diagnosed with AAV between 2000 and 2010 at Iwate Prefectural Central Hospital in Japan. Three GPA, two eosinophilic granulomatosis with polyangitis (EGPA) and three ANCA-associated classical polyarteritis nodosa patients were excluded based on the diagnostic criteria of the Research Group of Intractable Vasculitis of the Ministry of Health, Labor and Welfare of Japan (11). We also excluded one patient with propylthiouracil-induced MPA, four patients with active malignancies at the time of diagnosis, three receiving prednisolone prescribed by their previous physician and one with dementia who refused treatment. After excluding these 17 patients, 64 MPA patients ( 39 males and $25 \mathrm{fe}-$ males) were enrolled in this retrospective study. We followed up these patients for at least 6 months from the initiation of CS therapy or until death.

The patients were divided into two groups based on whether or not they received CY in addition to CS for remission induction during their first hospitalization. The patients were treated non-randomly, according to the judgment of the attending physician, either with a combination of $\mathrm{CY}$ and CS (CY group, n=29) or with CS alone (CS group, $n=$ $35)$.

\section{Clinical evaluation and outcome}

Clinical data at the time of diagnosis were obtained from hospital records. These data included the serum creatinine, albumin (Alb), C-reactive protein (CRP) levels, and the Birmingham vasculitis activity score 2003 (BVAS) values as confounding factors $(12,13)$. The estimated glomerular filtration rate was calculated according to the standard equation for Japanese patients (14). The primary outcome was all-cause mortality. The dates and causes of death were ob- tained from the patient records, including hospital and general practice records.

\section{Statistical analysis}

We compared the survival rate between the treatment groups using survival analysis. Furthermore, Kaplan-Meier curves were plotted and treatment groups were compared using the log-rank test. We calculated hazard ratios (HRs) using Cox regression hazard models. The following tests were used to compare the demographics and disease characteristics between treatment groups: chi-square test for categorical variables, Student's t-test for normally-distributed variables and the Mann-Whitney U-test for non-normally distributed variables. Data are shown as the mean \pm standard deviation or median (25-75th percentile). Statistical analyses were performed using the SPSS 17.0 software program (SPSS Inc., Chicago, IL), and $\mathrm{p}<0.05$ was considered to be statistically significant.

\section{Results}

\section{Patient characteristics}

The mean follow-up period was 38 months (range, 1-111 months) in the CY group and 41 months (range, 1-94 months) in the CS group. Table 1 shows the baseline characteristics of patients with ANCA-associated MPA. Mean ages in the CY and CS groups were $68 \pm 10$ and $69 \pm 13$ years, respectively. The proportion of male patients was higher in the CY group than in the CS group (76\% vs. $49 \%$, $\mathrm{p}=0.026)$. Four patients $(14 \%)$ in the $\mathrm{CY}$ group and three (9\%) in the CS group showed renal-limited vasculitis. The prevalence of MPO-ANCA-associated MPA was high in both groups: 24 patients $(83 \%)$ in the CY group and 33 (94\%) in the CS group. The number of patients with PR3ANCA-associated MPA was only five $(17 \%)$ in the CY group and two $(6 \%)$ in the CS group $(p=0.141)$. None of the patients were positive for both MPO-ANCA and PR3ANCA. There were no differences in the BVAS values and serum Alb levels between the two groups. The median serum creatinine level was slightly higher in the CY group than in the CS group, however, the difference was not significant $(2.7 \mathrm{mg} / \mathrm{dL}$ vs. $1.9 \mathrm{mg} / \mathrm{dL}, \mathrm{p}=0.492)$.

Among the total number of patients, renal involvement was observed in more than $90 \%$ patients and chest involvement in about $65 \%$ of patients (Fig. 1). Although nervous system involvement was slightly higher in the CS group than in the CY group, there was no statistically significant difference in the prevalence of organ involvement between the two groups.

\section{Treatment}

All patients received oral prednisolone (PSL) daily with or without an intravenous pulse dose of methylprednisolone (mPSL) of 250-1,000 mg daily for 3 consecutive days. Sixteen patients $(55.2 \%)$ in the CY group and $21(60.0 \%)$ in 
Table 1. Characteristics of the Patients at Baseline

\begin{tabular}{|c|c|c|c|c|}
\hline Characteristic & $\begin{array}{c}\text { All patients } \\
n=64\end{array}$ & $\begin{array}{c}\text { CY group } \\
\mathrm{n}=29\end{array}$ & $\begin{array}{c}\text { CS group } \\
n=35\end{array}$ & $\mathrm{p}$ value \\
\hline Mean age, $\mathrm{y}$ & $69 \pm 12$ & $68 \pm 10$ & $69 \pm 13$ & 0.653 \\
\hline Male, n (\%) & $39(61)$ & $22(76)$ & $17(49)$ & $0.026^{*}$ \\
\hline Body weight, kg & $54.8 \pm 9.4$ & $55.9 \pm 7.1$ & $53.9 \pm 11.0$ & 0.404 \\
\hline \multicolumn{5}{|l|}{ Diagnosis, $\mathrm{n}(\%)$} \\
\hline Microscopic polyangiitis & $57(89)$ & $25(86)$ & $32(91)$ & 0.505 \\
\hline Renal limited vasculitis & $7(11)$ & $4(14)$ & $3(9)$ & 0.505 \\
\hline Confirmatory renal biopsy, $\mathrm{n}(\%)$ & $41(61)$ & $22(76)$ & $19(54)$ & 0.073 \\
\hline MPO-ANCA-positive, n (\%) & $57(89)$ & $19(83)$ & $37(94)$ & 0.115 \\
\hline PR3-ANCA-positive, n (\%) & $7(11)$ & $5(17)$ & $2(6)$ & 0.115 \\
\hline Mean BVAS & $18.4 \pm 6.9$ & $18.2 \pm 5.8$ & $18.5 \pm 7.7$ & 0.863 \\
\hline Mean serum albumin level, $\mathrm{g} / \mathrm{dL}$ & $3.0 \pm 0.6$ & $2.9 \pm 0.7$ & $3.1 \pm 0.5$ & 0.388 \\
\hline Median serum creatinine level, mg/dL & $2.3(1.0-4.1)$ & $2.7(1.4-4.1)$ & $1.9(0.9-4.1)$ & 0.492 \\
\hline Median estimated GFR, $\mathrm{mL} / \mathrm{min}$ per $1.73 \mathrm{~m}^{2}$ & $20.5(11.1-48.0)$ & $19.7(11.6-41.1)$ & $23.3(10.0-62.2)$ & 0.642 \\
\hline Median CRP level, mg/dL & $4.15(0.39-12.13)$ & $3.8(0.4-11.3)$ & $4.5(0.6-13.9)$ & 0.787 \\
\hline
\end{tabular}

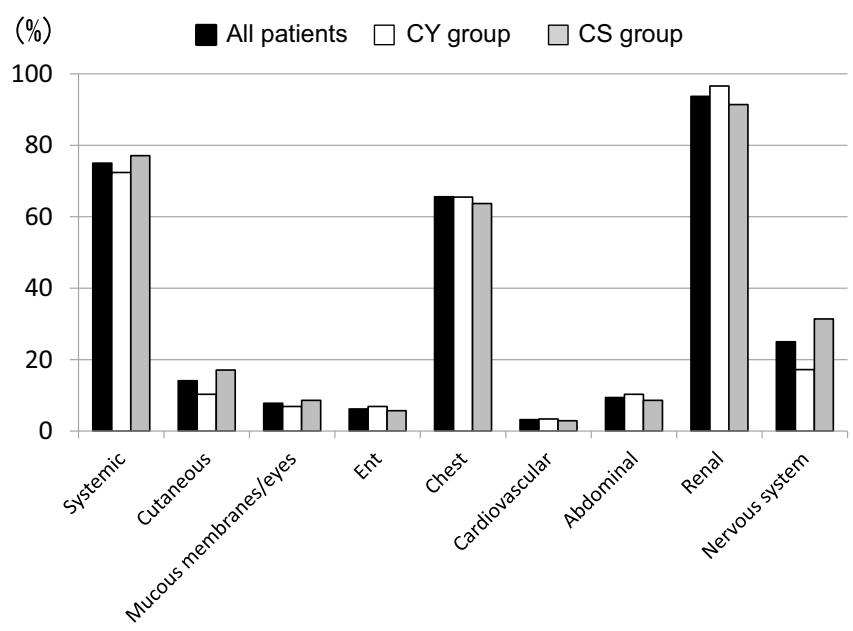

Figure 1. Prevalent organ involvement of the Birmingham vasculitis activity score 2003 (BVAS) in each group. There was no statistically significant difference in the prevalence of organ involvement between the two groups, although nervous system involvement was slightly higher in the CS group than in the $\mathrm{CY}$ group.

the CS group ( $\mathrm{p}=0.791)$ received pulse doses of mPSL. The average dosages of PSL in both groups were approximately equal at the initiation of treatment: $31.2 \pm 7.0 \mathrm{mg} / \mathrm{day}$ in the CY group and $30.1 \pm 10.8 \mathrm{mg} /$ day in the CS group, $\mathrm{p}=0.650$. The dosages of PSL in both groups were tapered at a similar rate for 1 month $(23.6 \pm 6.6$ and $22.3 \pm 7.0 \mathrm{mg} /$ day, respectively). However, the dosage of PSL at six months was smaller in the CY group than in the CS group (10.8 \pm 2.2 vs. $14.2 \pm 5.1 \mathrm{mg} /$ day; $\mathrm{p}=0.006$ ).

In the CY group, five patients were treated with oral $\mathrm{CY}$ at a dosage of $50 \mathrm{mg} /$ day, 16 with $25 \mathrm{mg} /$ day, and four with $25 \mathrm{mg}$ every other day. In three patients, oral CY was discontinued because two patients showed bone marrow suppression and one patient developed end-stage renal disease (ESRD) within a month. The median duration of oral $\mathrm{CY}$ treatment was 6 months and the maximum duration was 22 months. Intravenous (IV) CY (500 mg/month) pulse therapies were administered for two to six months in only four patients. CY was administered at various times after CS administration; the median interval from the initiation of CS to the initiation of $\mathrm{CY}$ was 18 days (range, 0-40 days). Fig. 2 shows age, body weight (BW) and eGFR for each dosage of oral CY and IVCY. The patients treated with lesser dosages of oral CY tended to be older, lighter weight, and associated with lower eGFR. IVCY therapy was done in younger and heavier patients with a low eGFR. The median levels of age, BW and eGFR in each group were as follows: 78 years old, $47.9 \mathrm{~kg}$, and $11.4 \mathrm{~mL} / \mathrm{min} / 1.73 \mathrm{~m}^{2}$ in patients with oral $\mathrm{CY}$ $25 \mathrm{mg}$ every other day $(\mathrm{n}=4), 67$ years old, $54.5 \mathrm{~kg}$, and $20.0 \mathrm{~mL} / \mathrm{min} / 1.73 \mathrm{~m}^{2}$ in those with oral CY $25 \mathrm{mg} /$ day $(\mathrm{n}=$ 16), 75 years old, $60.1 \mathrm{~kg}$, and $39.8 \mathrm{~mL} / \mathrm{min} / 1.73 \mathrm{~m}^{2}$ in those with oral CY $50 \mathrm{mg} /$ day $(\mathrm{n}=5), 63.5$ years old, 59.2 $\mathrm{kg}$, and $10.2 \mathrm{~mL} / \mathrm{min} / 1.73 \mathrm{~m}^{2}$ in those with $\operatorname{IVCY}(\mathrm{n}=4)$. Regarding the maintenance therapy for the CY group, only mizoribine was coadministered with CS in 2 patients. In the CS group, CY, mizoribine, cyclosporine, and methotrexate were coadministered with CS in 6, 2, one, and one patients, respectively. To prevent Pneumocystis jiroveci pneumonia (PJP), trimethoprim-sulfamethoxazole was used in 58.6\% (17 out of 29) in the CY group and $45.6 \%$ (16 out of 35 ) in the CS group ( $\mathrm{p}=0.303$; data not shown).

\section{Outcome and risk factors}

As a result of the induction therapy, 25 out of 29 patients (86.2\%) and 32 out of 35 (91.4\%) in CY and CS groups achieved remission, respectively; the difference was not significant ( $\mathrm{p}=0.527$; Fig. 3a). Among the total of 64 MPA patients in both groups, 22 died, including nine of $29(31 \%)$ in the CY group and 13 of $35(37 \%)$ in the CS group. In the $\mathrm{CY}$ group, the causes of death were pulmonary hemorrhage in 1 patient, gastrointestinal hemorrhage in 1, malignant disease in 2 , cardiovascular disease in 2 , bacterial pneumonia 
a

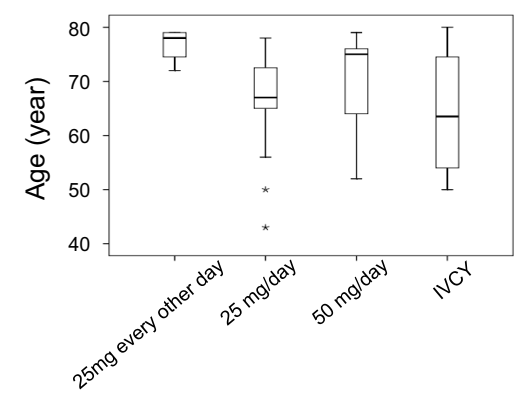

C

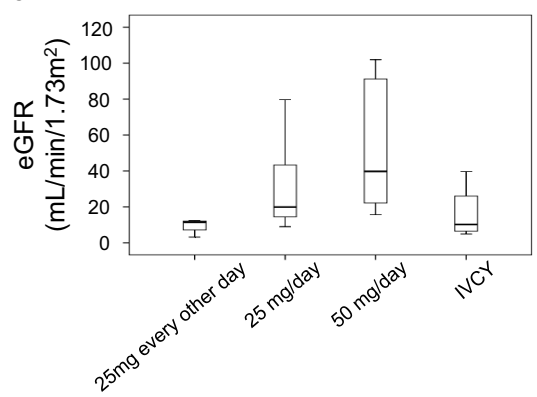

b

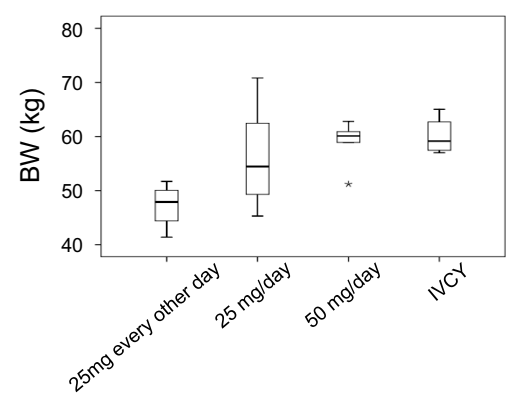

Figure 2. Age, body weight and eGFR for each dose of CY. (a) The median age levels were 78 years old in patients with oral CY $25 \mathrm{mg}$ every other day $(n=4), 67$ years old in those with oral CY $25 \mathrm{mg} /$ day $(\mathrm{n}=16)$, 75 years old in those with oral CY 50mg/day $(\mathrm{n}=5)$, and 63.5 years old in those with IVCY (n=4). (b) The median body weight levels were $47.9 \mathrm{~kg}, 54.5 \mathrm{~kg}, 60.1 \mathrm{~kg}$, and $59.2 \mathrm{~kg}$, respectively. (c) The median eGFR levels were $11.4 \mathrm{~mL} / \mathrm{min} / 1.73 \mathrm{~m}^{2}, 20.0 \mathrm{~mL} / \mathrm{min} / 1.73 \mathrm{~m}^{2} 39.8 \mathrm{~mL} /$ $\mathrm{min} / 1.73 \mathrm{~m}^{2}$, and $10.2 \mathrm{~mL} / \mathrm{min} / 1.73 \mathrm{~m}^{2}$, respectively.

in 1, PJP in 1, and Aspergillus pneumonia in 1 patient. In the CS group, the causes of death were pulmonary hemorrhage in 1 patient, uremia in 1 , gastrointestinal hemorrhage in 2 , bacterial pneumonia in 3 , PJP in 2 , bacteremia in 2 , and unknown causes in 2 which were likely related to physical deterioration associated with old age. The number of deaths due to infections was smaller in the CY group than in the CS group (three in the CY group and seven in the CS group). The infectious disease mortality rate at five years was $17 \%$ in the CY group and $23 \%$ in the CS group, although no statistical difference was found between the groups $(p=0.367)$. This trend was seen even at 6 months after the initiation of therapy, when most patients in the CY group were receiving CY (Table 2). Seventeen patients became dependent on hemodialysis, including seven (24\%) in the CY group and nine (26\%) in the CS group. Although eight patients needed hemodialysis on admission, fortunately the renal function recovered within a short period of time in two of these patients.

The survival rates at 1 and 5 years for all patients were 0.81 and 0.68 , respectively (Fig. $3 b$ ). The survival rate was slightly higher in the CY group than in the CS group, but there was no statistically significant difference (Fig. 3c; 0.86 vs. 0.77 at 1 year, 0.73 vs. 0.64 at 5 years, $\mathrm{p}=0.648$ ). Age, BVAS values, CRP levels and serum Alb levels were found to be related to the primary outcome by univariate analysis (Table 3a); however, sex, serum creatinine and ANCA type were not related to the primary outcome. Among patients receiving CY, the unadjusted HR was 0.813 and the HR after adjustment for age and sex was 0.710 (Table 3b). Although the HR decreased to 0.657 after full adjustment for age, sex, BVAS values, serum Alb levels and CRP levels, there was no statistically significant difference $(\mathrm{p}=0.386)$. In this final multivariate model, no factors were found to be independent risks. When we used the combination of ESRD and allcause mortality as an outcome, the survival rates were also not different between the two groups $(0.83$ vs. 0.69 at 1 year and 0.50 vs. 0.55 at 5 years, $p=0.963$; Fig. $3 d$ ).

\section{Discussion}

In this study, we were unable to find any statistically significant efficacy of the combination of CS and CY for remission induction in Japanese patients with ANCA-positive MPA, but the fully adjusted HR for mortality showed a tendency for decreased mortality rate in CY-treated patients (Table 3; HR=0.657). The efficacy of CY in AAV has been confirmed in several studies in the US and Europe (15-17). Hogan et al. reported that the non-adjusted HR for death was 0.18 among MPA patients treated with CY (18). CY was used in approximately half of the Japanese MPA patients in a Japanese prospective cohort study (JMAAV study) and a high remission rate was reported (19). However, in Japan, CY is not generally used in the treatment of cases of RPGN including AAV for remission induction (8). What is the reason for this difference between the West and Japan? Our findings suggest the following three possible explanations: First, there may exist ethnic and/or inherited dif- 
a
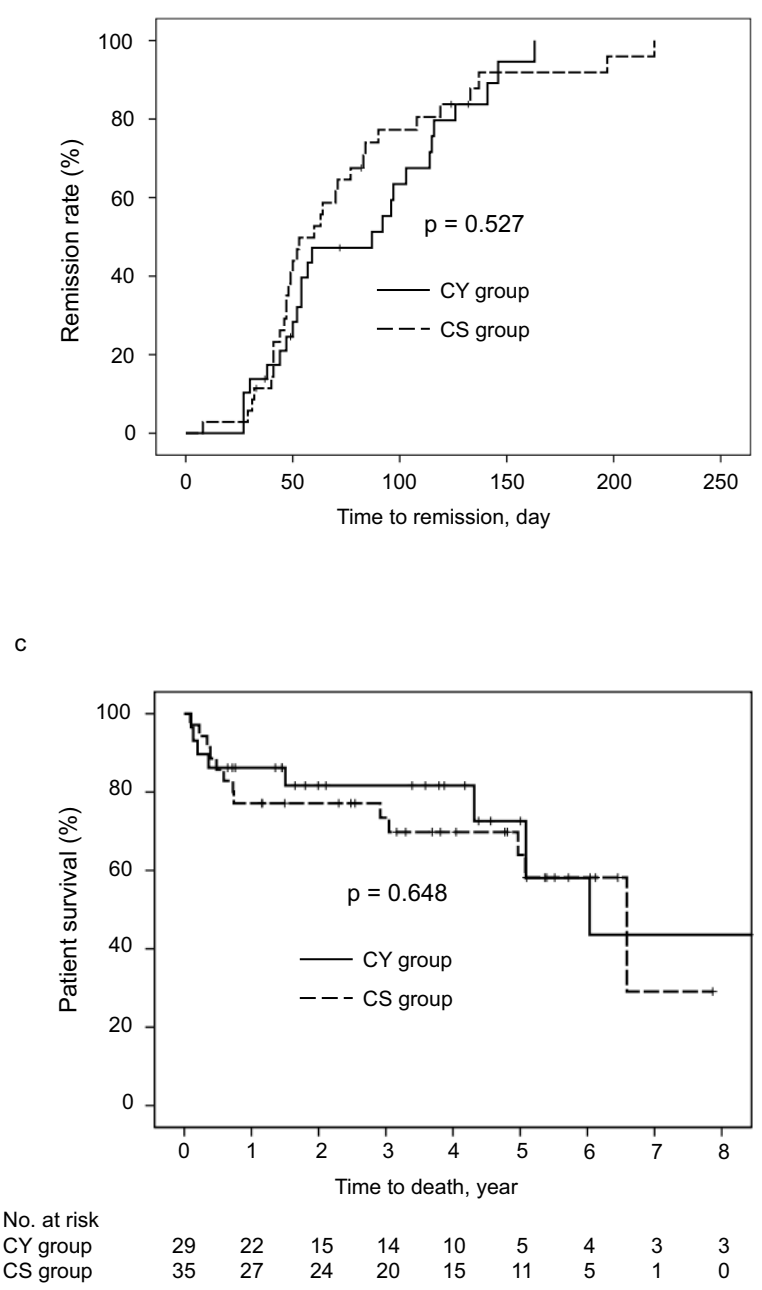

b

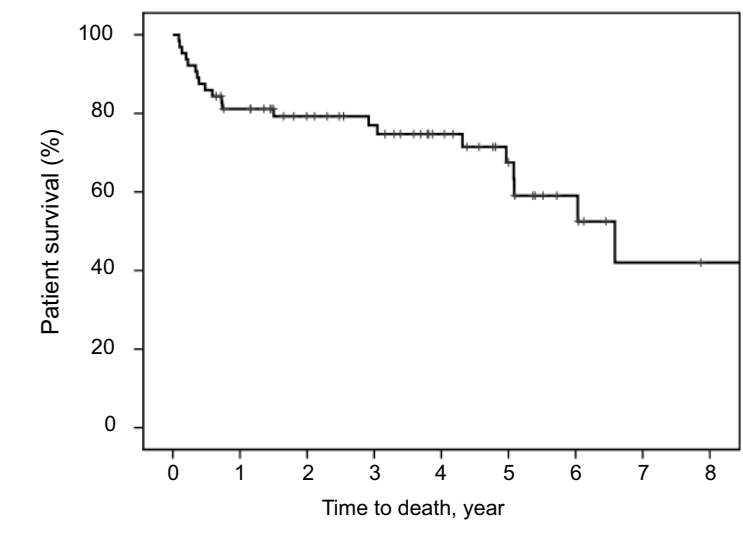

$\begin{array}{llllllllll}\text { No. at risk } & 64 & 49 & 39 & 34 & 25 & 16 & 9 & 4 & 3\end{array}$

d

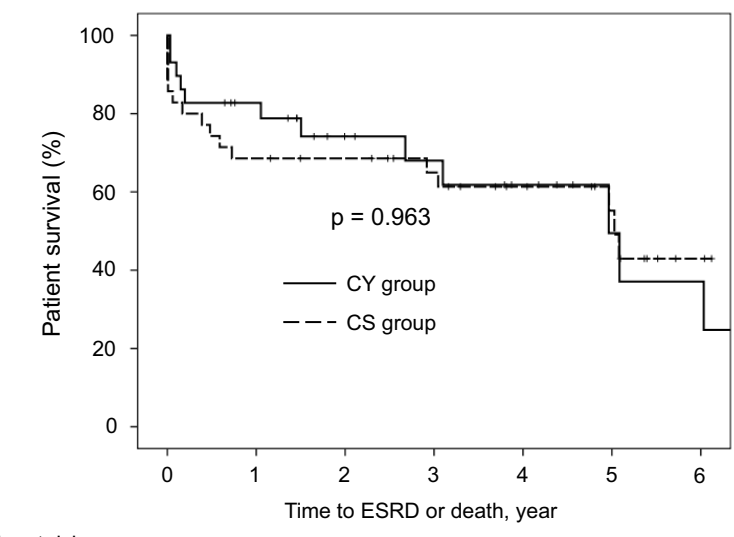

No. at risk

CY group

CS group

$\begin{array}{ccccccc}29 & 21 & 13 & 11 & 8 & 4 & 3 \\ 35 & 24 & 22 & 18 & 13 & 9 & 2\end{array}$

Figure 3. a. Kaplan-Meier curve for the remission of the CY group compared with the CS group. Remission rates were not substantially different between the two groups $(p=0.527)$. b. Survival curve for all patients. The survival rate for all patients was 0.81 at 1 year and 0.68 at 5 years. c. Survival curves of the CY group compared with the CS group. The survival rate was slightly better in the CY group than in the CS group; however, the difference was not statistically significant ( 0.86 vs. 0.77 at 1 year, 0.73 vs. 0.64 at 5 years, $p=0.648)$. d. Survival curves of the combination of ESRD and death. The survival rates in both groups were approximately equal $(0.83$ vs. 0.69 at 1 year, 0.50 vs. 0.55 at 5 years, $p=0.963$ ).

ferences between the two populations in response to immunosuppressive agents. Second, GPA in young patients, which responds well to immunosuppressive agents, constitutes most cases of AAV in Caucasians (5). In Japan, however, GPA is a rare disease whereas MPO-ANCA-associated MPA is the most common form of AAV in elderly patients. In particular, MPA in patients older than 60 years constitutes more than half of all Japanese cases of AAV, and as a result, the risk of infection due to CY therapy may be increased in Japanese AAV (8). Third, the Japanese guidelines for ANCA-positive RPGN are based on a Japanese nationwide retrospective survey which included all types of RPGN, and patients with AAV constituted about half of all the enrolled participants (8). To date however, there has been no Japanese nationwide survey investigating the efficacy of $\mathrm{CY}$ in Japanese AAV patients.
The number of early deaths directly associated with ANCA-positive MPA such as those due to alveolar hemorrhage was very small in both groups and short to long outcomes were good (Table 2). This study, in which the followup rate of patients was $98 \%$, also clarified the long-term outcome and causes of death in Japanese patients with ANCA-positive MPA. The number of deaths due to infection in the present study accounted for about half of allcause mortality, similar to the results of previous studies $(8,9,20)$. The number of deaths due to infection was less in the CY group than in the CS group, although no statistically significant difference was observed between the two groups. This might be related to the fact that the utilization rate of trimethoprim-sulfamethoxazole was higher and the PSL dosages at 6 months after the initial treatment were lower in the CY group than in the CS group. 
Table 2. Main Outcome Parameters according to Treatment Group

\begin{tabular}{|c|c|c|c|c|c|c|c|c|c|c|c|c|c|c|}
\hline \multirow[b]{2}{*}{ Parameter } & \multicolumn{2}{|c|}{ Baseline } & \multicolumn{2}{|c|}{6 Months } & \multicolumn{2}{|c|}{1 year } & \multicolumn{2}{|c|}{2 years } & \multicolumn{2}{|c|}{4 years } & \multicolumn{2}{|c|}{6 years } & \multicolumn{2}{|c|}{8 years } \\
\hline & $\begin{array}{c}\mathrm{CY} \\
\text { group }\end{array}$ & $\begin{array}{c}\text { CS } \\
\text { group }\end{array}$ & $\begin{array}{c}\text { CY } \\
\text { group }\end{array}$ & $\begin{array}{c}\text { CS } \\
\text { group }\end{array}$ & $\begin{array}{c}\mathrm{CY} \\
\text { group }\end{array}$ & $\begin{array}{c}\text { CS } \\
\text { group }\end{array}$ & $\begin{array}{c}\text { CY } \\
\text { group }\end{array}$ & $\begin{array}{c}\text { CS } \\
\text { group }\end{array}$ & $\begin{array}{c}\text { CY } \\
\text { group }\end{array}$ & $\begin{array}{c}\text { CS } \\
\text { group }\end{array}$ & $\begin{array}{c}\mathrm{CY} \\
\text { group }\end{array}$ & $\begin{array}{l}\text { CS } \\
\text { group }\end{array}$ & $\begin{array}{c}\text { CY } \\
\text { group }\end{array}$ & $\begin{array}{c}\text { CS } \\
\text { group }\end{array}$ \\
\hline Patients at risk, $\mathrm{n}$ & 29 & 35 & 25 & 30 & 22 & 27 & 15 & 24 & 10 & 15 & 4 & 5 & 3 & 0 \\
\hline All deaths, $\mathrm{n}$ & 0 & 0 & 4 & 5 & 4 & 8 & 5 & 8 & 5 & 10 & 7 & 12 & 8 & 13 \\
\hline Deaths from infections, $\mathrm{n}$ & 0 & 0 & 2 & 3 & 2 & 4 & 2 & 4 & 2 & 5 & 3 & 6 & 3 & 7 \\
\hline Deaths from others, $n$ & 0 & 0 & 2 & 2 & 2 & 4 & 3 & 4 & 3 & 5 & 4 & 6 & 5 & 6 \\
\hline End-stage renal disease, $\mathrm{n}$ & 1 & 5 & 3 & 7 & 3 & 8 & 4 & 8 & 6 & 8 & 7 & 9 & 7 & 9 \\
\hline Lost to follow-up, $\mathrm{n}$ & 0 & 0 & 0 & 0 & 0 & 0 & 0 & 1 & 0 & 1 & 0 & 1 & 0 & 1 \\
\hline
\end{tabular}

CY: cyclophosphamide, CS: corticosteroid

Table 3a. Hazard Ratios for the Primary Outcome Based on a Univariate Analysis

\begin{tabular}{lccc}
\hline & Hazard ratio & $95 \%$ confidence interval & p value \\
\hline Age ( per 1 year) & 1.048 & $1.003-1.095$ & $0.037^{*}$ \\
Sex (male) & 1.594 & $0.636-3.994$ & 0.609 \\
ANCA type (PR3) & 1.064 & $0.246-4.606$ & 0.934 \\
BVAS (per 1) & 1.073 & $1.012-1.136$ & $0.017^{*}$ \\
Serum albumin (per 1 g/dL) & 0.468 & $0220-0.996$ & $0.049^{*}$ \\
CRP (per 1 mg/dL) & 1.055 & $1.001-1.112$ & $0.047^{*}$ \\
Serum creatinine (per 1mg/dL) & 1.058 & $0.928-1.206$ & 0.399 \\
\hline
\end{tabular}

ANCA: anti-neutrophil cytoplasmic antibody, PR3: proteinase-3, BVAS: Birmingham

vasculitis activity score. ${ }^{*} \mathrm{p}<0.05$.

Table 3b. Hazard Ratios for the Primary Outcome Based on a Multivariable Analysis

\begin{tabular}{lccc}
\hline & Hazard ratio & $95 \%$ confidence interval & p value \\
\hline $\begin{array}{l}\text { CY } \\
\text { Unadjusted }\end{array}$ & 0.813 & $0.335-1.974$ & 0.648 \\
CY & 0.710 & $0.283-1.784$ & 0.467 \\
$\begin{array}{l}\text { adjusted by age and sex } \\
\text { CY }\end{array}$ & 0.722 & $0.287-1.813$ & 0.488 \\
$\begin{array}{l}\text { adjusted by age, sex, and BVAS } \\
\text { CY } \\
\text { adjusted by age, sex, BVAS, Serum albumin, CRP }\end{array}$ & $0.254-1.699$ & 0.386 \\
\hline \begin{tabular}{l} 
CY: cyclophosphamide, BVAS: Birmingham vasculitis activity score \\
\hline
\end{tabular}
\end{tabular}

The dosage of CY in this study was approximately half of the dosage recommended by the European League Against Rheumatism (EULAR) (15), since the recommended dosages are not considered to be appropriate for Japanese patients. The complete recommended dosage likely causes bone marrow suppression in Japanese MPA patients who are older and have lower eGFR just like the participants in the present study, compared with European participants in the previous study (6) (69 vs. 57 years old and 20.5 vs. 36.5 $\mathrm{mL} / \mathrm{min} / 1.73 \mathrm{~m}^{2}$, respectively), and Japanese elderly persons are of small stature. Although the serum creatinine levels were similar to those of the European study, the eGFR levels were approximately half.

Rituximab which is an anti-CD20 monoclonal antibody and mycophenolate mofetil have been recently reported to be efficacious in the treatment of AAV (21-24). However, the reported efficacy of these drugs is not superior to combined CS and CY therapy. Plasma exchange has also been reported to be more effective than intravenous methylprednisolone for the renal involvement of AAV (25). In Japan, however, plasma exchange is not common because the treatment for AAV is not covered by medical insurance (9). Hence, the options for remission induction therapy for AAV patients in Japan are limited to CS alone or the combination of CS and CY.

Two limitations in the present study need to be addressed. The first limitation concerns the treatment bias. The CY group might contain patients who did not respond to CS alone and, consequently were started on CY. The second one is a matter of CY dosages. Our patients received lower dosages of CY than those recommended by the EULAR, although we thought these dosages were sufficient as mentioned above. Therefore, there is the possibility that this may lead to an insignificant effect of CY on the outcome.

In conclusion, combination therapy with $\mathrm{CY}$ and CS tended to reduce the total number of deaths, but we could 
not find any statistically significant difference between the two groups. This study does not necessarily show that CY is ineffective for Japanese patients with ANCA-positive MPA, instead our findings suggest that $\mathrm{CY}$ may have a beneficial effect on some Japanese patients with ANCA-positive MPA. However, this study could not clarify what kind of patients benefit from combination therapy with CY and CS. Further studies, such as a prospective study or a randomized control study, are therefore needed to clarify this point.

The authors state that they have no Conflict of Interest (COI).

\section{References}

1. Jennette JC, Falk RJ, Bacon PA, et al. 2012 revised International Chapel Hill Consensus Conference Nomenclature of Vasculitides. Arthritis Rheum 65: 1-11, 2013.

2. Fujimoto S, Uezono S, Hisanaga $S$, et al. Incidence of ANCAassociated primary renal vasculitis in the Miyazaki Prefecture: the first population-based, retrospective, epidemiologic survey in Japan. Clin J Am Soc Nephrol 1: 1016-1022, 2006.

3. Watts RA, Scott DG, Jayne DR, et al. Renal vasculitis in Japan and the UK--are there differences in epidemiology and clinical phenotype? Nephrol Dial Transplant 23: 3928-3931, 2008.

4. Fujimoto S, Watts RA, Kobayashi S, et al. Comparison of the epidemiology of anti-neutrophil cytoplasmic antibody-associated vasculitis between Japan and the U.K. Rheumatology (Oxford) 50 1916-1920, 2011

5. Fauci AS, Katz P, Haynes BF, Wolff SM. Cyclophosphamide therapy of severe systemic necrotizing vasculitis. N Engl J Med 301 235-238, 1979.

6. de Groot K, Harper L, Jayne DR, et al. Pulse versus daily oral cyclophosphamide for induction of remission in antineutrophil cytoplasmic antibody-associated vasculitis: a randomized trial. Ann Intern Med 150: 670-680, 2009.

7. Harper L, Morgan MD, Walsh M, et al. Pulse versus daily oral cyclophosphamide for induction of remission in ANCA-associated vasculitis: long-term follow-up. Ann Rheum Dis 71: 955-960, 2012.

8. Koyama A, Yamagata K, Makino H, et al. A nationwide survey of rapidly progressive glomerulonephritis in Japan: etiology, prognosis and treatment diversity. Clin Exp Nephrol 13: 633-650, 2009.

9. Guidelines for the treatment of rapidly progressive glomerulonephritis, second version. Nihon Jinzo Gakkai Shi 53: 509-555, 2011 (Abstract in English).

10. Ethical Guidelines for Epidemological Research. 2002 (Accessed at http://www.niph.go.jp/wadai/ekigakurinri/guidelines.pdf).

11. Ozaki S. ANCA-associated vasculitis: diagnostic and therapeutic strategy. Allergol Int 56: 87-96, 2007.

12. Luqmani RA, Bacon PA, Moots RJ, et al. Birmingham Vasculitis Activity Score (BVAS) in systemic necrotizing vasculitis. QJM 87: 671-678, 1994.

13. Flossmann O, Bacon P, de Groot K, et al. Development of comprehensive disease assessment in systemic vasculitis. Ann Rheum Dis 66: 283-292, 2007.

14. Matsuo S, Imai E, Horio M, et al. Revised equations for estimated GFR from serum creatinine in Japan. Am J Kidney Dis 53: 982992, 2009.

15. Mukhtyar C, Guillevin L, Cid MC, et al. EULAR recommendations for the management of primary small and medium vessel vasculitis. Ann Rheum Dis 68: 310-317, 2009.

16. Adu D, Pall A, Luqmani RA, et al. Controlled trial of pulse versus continuous prednisolone and cyclophosphamide in the treatment of systemic vasculitis. QJM 90: 401-409, 1997.

17. de Groot K, Adu D, Savage CO; Euvas. The value of pulse cyclophosphamide in ANCA-associated vasculitis: meta-analysis and critical review. Nephrol Dial Transplant 16: 2018-2027, 2001.

18. Hogan SL, Nachman PH, Wilkman AS, Jennette JC, Falk RJ. Prognostic markers in patients with antineutrophil cytoplasmic autoantibody-associated microscopic polyangiitis and glomerulonephritis. J Am Soc Nephrol 7: 23-32, 1996.

19. Ozaki S, Atsumi T, Hayashi T, et al. Severity-based treatment for Japanese patients with MPO-ANCA-associated vasculitis: the JMAAV study. Mod Rheumatol 22: 394-404, 2012.

20. Turnbull J, Harper L. Adverse effects of therapy for ANCAassociated vasculitis. Best Pract Res Clin Rheumatol 23: 391-401, 2009.

21. Stone JH, Merkel PA, Spiera R, et al. Rituximab versus cyclophosphamide for ANCA-associated vasculitis. N Engl J Med 363: 221-232, 2010.

22. Jones RB, Cohen Tervaert JW, Hauser T, et al. Rituximab versus cyclophosphamide in ANCA-associated renal vasculitis. N Engl $\mathrm{J}$ Med 363: 211-220, 2010.

23. Silva F, Specks U, Kalra S, et al. Mycophenolate mofetil for induction and maintenance of remission in microscopic polyangiitis with mild to moderate renal involvement--a prospective, openlabel pilot trial. Clin J Am Soc Nephrol 5: 445-453, 2010.

24. Hu W, Liu C, Xie H, Chen H, Liu Z, Li L. Mycophenolate mofetil versus cyclophosphamide for inducing remission of ANCA vasculitis with moderate renal involvement. Nephrol Dial Transplant 23: 1307-1312, 2008.

25. Jayne DR, Gaskin G, Rasmussen N, et al. Randomized trial of plasma exchange or high-dosage methylprednisolone as adjunctive therapy for severe renal vasculitis. J Am Soc Nephrol 18: 21802188, 2007.

(C) 2013 The Japanese Society of Internal Medicine http://www.naika.or.jp/imonline/index.html 